\title{
Autonomic function test in chronic renal failure patients.
}

\author{
Deivanayagame $B^{*}$ \\ Department of Physiology, Sri Lakshmi Narayana Institute of Medical Sciences, Osudu Agaram, Villianur Commune, \\ Puducherry, India
}

\begin{abstract}
Background: Autonomic dysfunction has been recognized as an important contributor to the poor outcome in chronic kidney disease patients. Several studies have reported abnormalities in autonomic function tests in chronic renal failure.

Objectives: To study the effect of chronic renal failure on autonomic function tests and to compare the difference in autonomic function test between normal versus chronic renal failure patients,

Material and methods: This is a comparative case control study undertaken by me in the Department of physiology, cardiovascular autonomic function tests were performed for Thirty cases of chronic renal failure patients and thirty matched controls of both the genders after obtaining informed written consent and then compared, which include deep breathing test, valsalva maneuver, diastolic pressure response to cold pressor test, response to standing on heart rate and hand grip test.

Result: A total of 60 respondent are available for data analysis and the normal healthy subjects values are correlated with the chronic renal failure patients, for valsalva ratio $(p=0.0001)$, deep breathing test $(p=0.0003)$, response to standing in heart rate $(p=0.0124)$ rise in diastolic blood pressure in hand grip test $(p=0.001)$ and rise in diastolic Blood pressure in cold pressor test $p=0.001)$ respectively.

Conclusion: This study concludes both parasympathetic and sympathetic dysfunction is seen chronic renal failure patients.
\end{abstract}

Keywords: Chronic renal failure, Autonomic neuropathy, Cold pressor test, Uremia.

Accepted on May 15, 2019

\section{Introduction}

Chronic kidney disease encompasses a spectrum of different pathophysiological processes associated with abnormal kidney function, and a progressive decline in glomerular filtration rate. A widely accepted classification based on guidelines of national kidney foundation in which stages of CKD are defined according to the estimated GFR. The term chronic renal failure applies to the processes of continuing irreversible reduction in nephron number and typically corresponds to CKD stages 3-5. CKD stage 1and 2 CKD are usually not associated with any symptoms arising from the decrement in GFR. If the decline in GFR progresses to 3 and 4 clinical and laboratory complications of CKD become more prominent. If the patient progresses to CKD stage 5 toxins accumulates and eventuating in uremic syndrome and Before the advancement of modern medicine renal failure was referred to as Uremic poisoning. Uremia was the term used to describe the contamination of the blood with urine. Around 1847, this term was used to describe reduced urine output, now known as oliguria, which was thought to be caused by the urine mixing with blood instead of being voided through the urethra. Some of the common causes of chronic renal failure are chronic glomerulonephritis and polycystic kidney. Two major systemic diseases which frequently involve the kidneys and lead to CRF are hypertension and diabetes mellitus. Diabetes is a common cause of renal failure. Hypertensive nephropathy is a common cause of CKD in the elderly, in whom chronic renal ischemia as a result of small and large vessel renovascular diseases may be underdiagnosed [1]. The increased mortality rate is found to be associated with long term complications of diabetes [2,3]. Different mechanism through which cardiac autonomic nervous dysfunction promotes mortality includes myocardial ischemia, silent myocardial infarction impaired respiratory response to hypoxia, intraoperative cardiovascular liability, and fatal arrhythmia due to QT prolongation. However mechanisms remain unclear. Because Cardiac Autonomic Neuropathy is common in patients with diabetic nephropathy we evaluated the CAN in chronic renal failure patients.

\section{Materials and Methods}

This is a comparative case control study undertaken by me in the Department of physiology, cardiovascular autonomic function tests were performed for thirty cases of chronic renal failure patients and thirty matched controls of both the genders after obtaining informed written consent and then compared.

The criteria for selecting the cases are: 
1. Presence of chronic renal failure as determined by NKF/ DOQI 2002 guidelines [4].

2. Age between 30-70 years.

3. Patient with no $\mathrm{H} / \mathrm{o}$ recent myocardial infarction\& medically unstable patients.

4. No H/O Drugs like beta blocker, Diuretics, Nitrates, Tricyclic antidepressants alcohol for past 1 week.

All experiments were performed at the cardiac autonomic function research laboratory in Department of Physiology. The tests for the assessment of cardiovascular autonomic functions are as follows.

\section{Deep breathing test}

This test is used to assess the parasympathetic activity.

Principle: Heart rate increases during inspiration due to decreased cardiac vagal activity and decreases during expiration due to increased vagal activity. This is detected by recording lead-II E.C.G continuously when the subject is breathing deeply $[5,6]$.

Method: Subject was instructed to maintain deep breathing at a rate of six breaths per minute. Subject was made to lie down comfortably in supine position with head elevated to 300. ECG electrodes were connected for recording Lead II ECG continuously in Audacity software and the R-R interval was measured using the beat finder tool in the software. While subject was breathing deeply at a rate of 6 breaths per minute (allowing 5 seconds each for inspiration and expiration) maximum and minimum heart rates were recorded with each respiratory cycle. Expiration to inspiration ratio was determined by using the formula

$E: I$ ratio $=\frac{\text { Maximum } R-R \text { interval during deep expiration }}{\text { Minimum } R-R \text { interval during deep inspiration }}$

\section{Valsalva manoeuver}

The valsalva ratio is a measure of parasympathetic and sympathetic functions. For the response to occur in valsalva manoeuver parasympathetic acts as afferent and efferent and sympathetic acts as a part of the efferent pathway. Therefore the valsalva ratio assesses more of parasympathetic function $[5,7]$.

Method: Subject was made to lie down in a semi recumbent or sitting position. Nostrils were closed manually. Mouth piece was put into the mouth of the subject and the Mercury manometer was connected to the mouth piece. ECG machine was switched on for continuous recording. Subject was asked to exhale forcefully into the mercury manometer and asked to maintain the expiratory pressure at $40 \mathrm{~mm}$ of $\mathrm{Hg}$ for $10-15$ seconds. ECG changes were recorded throughout the procedure, 30 seconds before and after the procedure i.e., the ratio of the longest $\mathrm{R}-\mathrm{R}$ interval (which comes shortly after the release) to the shortest $\mathrm{R}-\mathrm{R}$ interval which occurs during maneuver.

Valsalva ratio was calculated by using the formula:

$$
\begin{aligned}
& \text { Valsalva }= \\
& \text { Longest } R-R \text { interval after the manoeuver }(\text { Phase }-I V) \\
& \text { Shortest } R-R \text { interval during the manoeuver }(\text { Phase }-I I)
\end{aligned}
$$

\section{Cold pressor test III (cold pressure test)}

The afferent limb of the reflex pathway in cold pressor test is somatic fibres whereas the efferent limb is sympathetic fibres $[5,7,8]$.

Principle: Submerging the limbs in cold water results in an increase in systolic and diastolic blood pressures, which is detected by sphygmomanometer.

Method: Subject was instructed regarding the test. Blood pressure was recorded under basal conditions. Cold water was taken in a container. Subject was asked to submerge one of his hand to his wrist in cold water. Blood pressure was recorded from the other hand at 30 seconds interval for a period of 2 minutes using automatic BP Monitor (Omron).

Heart rate response to standing: This test was conducted after 10 minutes of supine rest. Then the patient was told to stand within 3 seconds and BP and heart rate were recorded at baseline and at 2 nd minute 30:15 ratios were calculated as the ratio between longest $\mathrm{R}-\mathrm{R}$ at or around the 30th beat and shortest R-R at or around the 15 th beat.

\section{Hand grip test}

In the hand grip test, there is a rise in heart rate and blood pressure. The blood pressure rise is due to increased sympathetic activity and heart rate rise is due to decreased parasympathetic activity $[5,6]$.

Principle: Sustained hand grip against resistance causes an increase in heart rate and blood pressure. These responses are detected by using ECG and blood pressure monitors using electronic automatic B.P apparatus (Omron brand).

Method: Subject was instructed regarding the test, Subject was made to lie down in semi recumbent position. ECG electrodes were connected for lead II recording of ECG and sphygmomanometer for blood pressure measurement Basal heart rate and blood pressure were recorded, Subject was asked to maintain a pressure of $30 \%$ of the maximum voluntary contraction in the hand grip dynamometer for about $5 \mathrm{~min}$ with the dominant hand Heart rate and change in SBP, DBP were recorded every minute using Automatic BP monitor (Omron HEM 780) for measurement of Blood pressure.

Note: Change in DBP is defined as the difference between the last value recorded before the release of hand grip pressure and the resting DBP recorded before commencing isometric exercise. The normal response is rise in DBP by $>10-15 \mathrm{~mm}$ of $\mathrm{Hg}$ and rise in heart rate by about $30 \%$ of the pretest value.

The above mentioned Autonomic function tests were conducted in 30 chronic renal failure patients, and 30 healthy controls and the results were compared to study the Autonomic function test 


\section{Results}

The results of autonomic function test between the healthy normal control and chronic renal failure patient's age and sex distribution of the patient and control are depicted in the Table 1 .
As shown in Table 2, heart rate response to breathing, valsalva ratio, diastolic pressure during hand grip and cold pressor test and heart rate response to standing are significant between the patient and the healthy normal control.

Table 1. Demographic profile of the patient, values expressed in mean \pm standard deviation: both parameters are not significant between both groups.

\begin{tabular}{llcl}
\hline SI. No. & Parameters & CRF Patients & Control \\
\hline 1 & Age & $52.766 \pm 5.998$ & $52.8 \pm 3.717$ \\
\hline 2 & Gender (male:female) & $19: 11$ & $18: 12$ \\
\hline Values expressed in mean \pm standard deviation: both parameters are not significant between both groups \\
\hline
\end{tabular}

Table 2. Comparison of autonomic function test between control and CRF patients.

\begin{tabular}{|c|c|c|c|c|c|}
\hline SI No. & Test & Parameter & CRF Patients & Control & p-Value \\
\hline 1 & Deep breathing test & Delta heart rate & $1.072 \pm 0.096$ & $1.154 \pm 0.06$ & 0.0003 \\
\hline 2 & Valsalva maneuver & Valsalva ratio & $1.147 \pm 0.134$ & $1.24 \pm 0.1$ & 0.0001 \\
\hline 3 & Handgrip test & Rise in diastolic pressure & $6.333 \pm 2.928$ & $9.133 \pm 3.626$ & 0.001 \\
\hline 4 & Cold pressor test & Rise in diastolic pressure & $10.266 \pm 2.815$ & $13.8 \pm 3.907$ & 0.001 \\
\hline 5 & Response to standing & Heart rate & 7.052 & $3.733 \pm 3.687$ & 0.0124 \\
\hline
\end{tabular}

Values expressed in Mean \pm SD

\section{Discussion}

Comparison group in our study is between healthy volunteer and chronic renal failure patient.

Comparison between chronic renal failure patient and healthy control in deep breathing test showed statistically significant value ( $p$-value of 0.0003 ).

In valsalva maneuver, between control and chronic renal failure patients $\mathrm{p}$ value is 0.001 , which is significant.

Result obtained in control vs. CRF patients in heart rate response to standing is $\mathrm{p}=0.0124$ (Significant).

Comparison between two groups in diastolic blood pressure response to cold pressor test is $\mathrm{p}=0.001$ which is markedly significant.

Diastolic BP response to hand grip test showed significant lower values for chronic renal failure patients when compared to healthy individual.

Test to asses parasympathetic activity are deep breathing test, valsalva maneuver and heart rate response to standing and test to assess the sympathetic functions are diastolic BP response to cold pressor test and diastolic BP response to handgrip test. All the autonomic function test are abnormal in chronic renal failure patients when compared to normal healthy volunteers which suggests that there is both parasympathetic and sympathetic involvement are seen in CRF patients.
Our study is in accordance with Kamal et al. [9] who showed heart rate response to standing in uremic patient on standing and post standing after hemodialysis showed improvement. It may be because of decrease of level of chemical component in blood e.g. urea and creatinine. They concluded that uremia causes deterioration of autonomic function (heart rate response to standing).

Our study matches Autonomic function test done by Campese et al. [10] on normal vs. predialysis and dialysis patients. Using 3 tests valsalva ratio, handgrip exercise and response orthostats showed that ANS function disturbance are common in uremia and the mechanism behind that is reduced end organ response to norepinephrine.

Our study is in accordance with Study by Malik et al. [11]. His results showed both parasympathetic and sympathetic damage common in CRF independent of patient Irrespective of the treatment regimen the clinical sequele was the same.

Our study is also in accordance with Sanya et al. [12]

\section{Strength}

In this study we used 5 battery of test which are non-invasive which can be done easily by us, so that we can determine autonomic damage early and prevent the progression of the disease thereby prevent the cardiovascular morbidity. 


\section{Limitations}

In our study are less sample size are used and it is conducted for a short duration, and at same time severity of chronic renal failure are not taken into consideration in our study.

\section{Conclusion}

The autonomic function system integrity in chronic renal failure can be assessed by the autonomic function tests. As the population of Chronic Kidney disease is rapidly increasing autonomic function tests were done for these patients and after the study we found that chronic renal failure Patients were found to have combined autonomic failure. In this test whenever there is sympathetic dysfunction there is associated parasympathetic abnormality. From this test it is concluded that detection of autonomic neuropathy among patients with CKD in earlier stages may provide a mean to follow CKD progression.

\section{References}

1. Harrisonns principles of internal medicine (2nd edn.) McGraw Hill Co., United States 2019; pp 1761-1763.

2. Broscious DK, Castagnola J. Chronic kidney disease: Acute manifestations and role of critical care nurses. Crit Care Nurse 2006;26: 17-27.

3. Vinik AI, Maser RE, Mitchell BD, Freeman R. Diabetic autonomic neuropathy. Diabetes Care 2003;26: 1553-1579.

4. National Kidney Foundation. K/DOQI clinical practice guidelines for chronic kidney disease: Evaluation, classification, and stratification. Am J Kidney Dis 2002;40: 1-266.

5. Pal GK. Autonomic Function Tests: In: Text Book of Practical Physiology. Orient Longman, India 2009; pp 296-304.
6. Ewing DJ, Clarke BF. Autonomic neuropathy: Its diagnosis and prognosis. Clin Endocrinol Metab 1986;15: 855-887.

7. Ewing DJ, Clarke BF. Diagnosis and management of diabetic autonomic neuropathy. Br Med J Clin Res Ed 1982;285: 916-918.

8. Clarke BF, Ewing DJ. Cardiovascular Reflex Tests. In: The Natural History of Diabetic Autonomic Neuropathy. NY State J Med, USA 1982; pp 903-905.

9. Kamal A. Effect of hemodialysis on autonomic dysfunction in patients with chronic renal failure. Neurosciences 2000;5: 50-56.

10. Campese VM, Romoff MS, Levitan D, Lane K, Massry SG. Mechanisms of autonomic nervous system dysfunction in uremia. Kidney Int 1981;20: 246-253.

11. Malik S, Winney RJ, Ewing DJ. Chronic renal failure and cardiovascular autonomic function. Nephron 1986;43: 191-195.

12. Sanya EO, Wieling W, Mancia G. Blood pressure and heart rate variability in autonomic disorders: A critical review. Clin Auton Res 1996;6: 171-182.

\section{*Correspondence to}

Deivanayagame B

Department of Physiology

Sri Lakshmi Narayana Institute of Medical Sciences

Osudu Agaram, Villianur Commune

Puducherry

India 